\title{
Repurposing hypothyroidism drug liothyronine for cancer immunotherapy
}

\author{
Xiuman Zhou \\ Jiangfeng Du \\ Hongfei Wang \\ Chunxia Chen \\ Ling Jiao \\ Xiangrui Cheng \\ Xiaowen Zhou \\ Shaomeng Chen \\ Shanshan Gou \\ Wenshan Zhao \\ Wenjie Zhai \\ Junhui Chen \\ Yanfeng Gao Ph D
}

\section{Video Byte}

Keywords: Cell Communication and Signaling, PVR, TIGIT, small molecule compound, virtual screening, liothyronine, cancer immunotherapy, immune checkpoint blockade, CD8+, molecular docking, drug discovery, TIMER, Oncomine, GEO database, flow cytometry, MC38 tumor model, regulatory T cells, Tregs, colorectal cancer, NK cells

Posted Date: November 12th, 2020

DOl: https://doi.org/10.21203/rs.3.rs-106669/v1

License: (9) This work is licensed under a Creative Commons Attribution 4.0 International License. Read Full License 


\section{Abstract}

Immune checkpoint blockade has enabled great progress in cancer immunotherapy. TIGIT is a novel immune checkpoint that plays a critical role in cancer immunity. TIGIT's inhibitory function relies on the molecule's interaction with the ligand PVR. That makes blocking the TIGIT/PVR interaction a promising therapeutic approach. But small molecules suited for the job have not yet been investigated. Now, research points to the benefits of the molecule liothyronine. Liothyronine is an artificial hormone used to treat hypothyroidism. A combination of molecular docking, screening, and a series of in vitro and in vivo assays revealed that liothyronine could be a suitable TIGIT/PVR blocker. When applied to tumor-bearing mice, liothyronine significantly inhibited tumor growth by enhancing CD8+ T cell infiltration and immune responses. The structure-activity relationships and extensive side effects of liothyronine remain to be investigated but the findings demonstrate the power of molecular docking for the repurposing of approved drugs and, more importantly, point to a new potential candidate for cancer immunotherapy. 